\title{
Treatment of recto - urethral fistula: state of the art in brief
}

Volume 5 Issue 2 - 2017

\section{Opinion}

Recto-urethral fistulas (RUF) is a communication between the rectum and the bladder or the urethra. The causes can be divided in congenital or acquired. Congenital RUF is rare and it is caused by inflammatory bowel diseases or trauma, ${ }^{1}$ while acquired RUF is more frequent and is often caused by a rectal wall damage occurring during radical prostatectomy (RP) or less frequently during cryotherapy or radiotherapy. ${ }^{2}$ In case of RP the incidence of RUF ranges between 0.6 to $9 \%$ regardless the surgical approach. ${ }^{3,4}$ From the patho-physiology point of view the RUF is a communication between the lower part of the rectum and the urethra: in this context the higher pressure in the rectum kept by the normal sphinteric muscles contraction leads to the passage of air and later faeces from the rectum to the bladder causing pneumaturia, faecaluria, pelvic collection, urinary infection, fever and potentially sepsis. The treatment of this serious postsurgical complication is difficult for both urologist and surgeons obtaining a solution in a short time very rarely.

The diagnosis is composed by the clinical signs mentioned above and a radiological image, that frequently consist on a cystography (Figure 1). As first attempt to solve the fistula, the patients undergoes a longer urinary catheterization, medical therapy and closure of the rectal wall damage. Only up to $46 \%$ of RUF 5 obtain the closure with this so called "conservative treatment". In case of recurrent RUF, the first step is the reduction of the fistula "flow" through a double diversion: faecal diversion (colostomy) and urethral catheterization. At this point multiple approaches and surgical techniques have been proposed. Hechenbleikner EM et al. ${ }^{6}$ has shown that patients affected by RUF undergoes up to 4 categories of repair with a $87,5 \%$ rate of fistula closure and with more than $10 \%$ rate of permanent fecal and/or urinary diversion. Techniques available can divided in 2 groups: transabdominal (open or laparoscopic) and perineal. In the trans-abdominal group the linkage closure is obtained suturing the rectal wall lesion with or without omentum patch, clips or glue. Unfortunately results are poor with low rate of fistula solution.

In the perineal techniques group, the York-Mason procedure (trans rectal-trans sfinteric), the trans perineal fistula closure with the interposition of pedicled gracilis or of bulbocavernosus muscle and the trans anal fistula repair with rectal wall flap advancement have to be mentioned. Even if some authors have recently proposed the fistula suture trans-anally with laparoscopic devices ${ }^{7}$ to allow to work in a narrow space, the medium distance between the RUF and the anal margin is $6 \mathrm{~cm}$, a distance easily accessible with a Parks anal retractor, patient in a Jack-Knife position and instruments used in proctology.

The York-Mason operation consists on a paracoccigeal incision with the patient in a prone position and a subsequent posterior rectal wall opening that allow the exposure of the anterior wall. The RUF is than dissected and a plane with the bladder is created to ensure a tension-free closure. ${ }^{8}$ The interposition of bublo-cavernosus or gracilis muscles is a well known technique that aims to reinforce the fistula closure with autologous tissue with a trans-anal or trans-

\author{
Inama M,' Moretto $\mathrm{G}^{2}$ \\ 'PhD student at Bioengineering and Medical-Surgical Sciences, \\ Politecnico di Torino, Italy \\ ${ }^{2}$ General Surgery Unit, Hospital Dott Pederzoli, Italy
}

Correspondence: Marco Inama, General Surgery Unit, Hospital “Dott. Pederzoli” Via Monte Baldo 24, 37019 Peschiera del Garda, Verona, Italy, Tel 00390456449 319, Fax 0039045 6449 II5, Email inama.marco@gmail.com

Received: January 08, 2017| Published: August 03, 2017

perineal approach. ${ }^{9,10}$ The York-Mason and the autologous muscle interposition are techniques with a high risk of wound infections, injuries of neurovascular structures and urethral stenosis but with high rate of fistula closure.

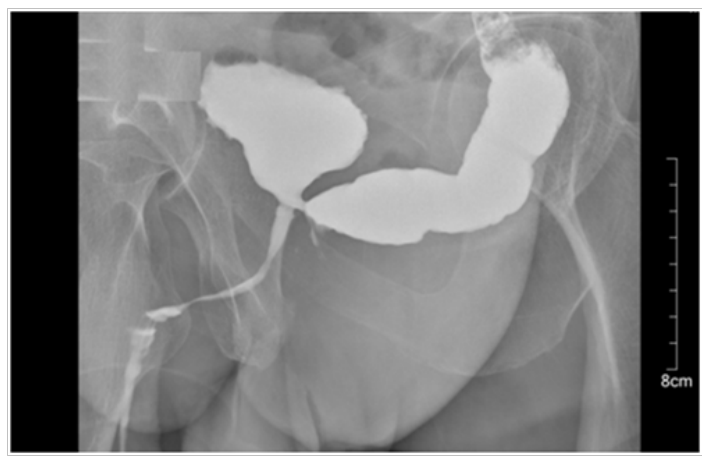

Figure I Cystography.

The trans-anal approach is the least commonly used, but it has the advantage of not involving the division of the anal sphincter. Some authors have recently reported their experience treating the RUF trans-anally with the use of biological mesh covered by a tension free-well vascularised rectal wall flap. ${ }^{11-13}$ Acellular dermal matrix (biological mesh) is potentially advantages to reduce risks of infection or rejection. Regardless the cause, position or dimension of the fistula, all RUF should undergo a conservative treatment. In case of recurrence, a double diversion has to be performed as first step, followed by the surgical repair of the fistula. Since there is no standardized treatment and knowledge's in this field are based on retrospective-single centre experiences with poor numbers of patients, the choice of the surgical technique is usually determined by the surgeon's familiarity with a given procedure. Comparative studies are warranted to better elucidate the best clinical and surgical treatment in case of chronic-persistent recto-urethral fistula. 


\section{Acknowledgments}

None.

\section{Conflicts of Inetrset}

None.

\section{References}

1. Shah TT, Ahmed H, Kanthabalan A, et al. Focal cryotherapy of localized prostate cancer: a systematic review of the literature. Expert Rev Anticancer Ther. 2014;14(11):1337-1347.

2. Beddy D, Poskus T, Umbreit E, et al. Impact of radiotherapy on surgical repair and outcome in patients with rectourethral fistula. Colorectal Dis. 2013;15(12):1515-1520.

3. Kitamura H, Tsukamoto $\mathrm{T}$. Rectiurinary fistula after radical prostatectomy: review of the literature for incidence, aetiology and management. Prostate Cancer. 2011:629105.

4. Novara G, Ficarra V, Rosen RC, et al. Systematic Review and metanalysis of perioperative outcomes and complications after robot-assisted radical prostatectomy. Eur Urol. 2012;62(3):431-452.

5. al-Ali M, Kashmoula D, Saoud IJ. Experience with 30 posttraumatic rectourethral fistula: presentation of posterior trans-sphinteric anterior rectal wall advancement. J Urol. 1997;158:421-424.
6. Hechenbleikner EM, Buckley JC, Wick EC. Acquired rectourethral fistulas in adults: a systematic review of surgical repair techniques and outcomes. Dis Colon Rectum. 2013;56(3):374-383.

7. Nicita G, Villari D, Caroassai S, et al. Minimally invasive transanal repair of rectourethral fistulas. Eur Urol. 2017;71(1):133-138.

8. Kasraeian A, Rozet F, Cathelineau X, et al. Modified York Mason technique for repair of iatrogenic rectourinary fistula: the Montsouris experience. J Urol. 12019;181(3):1178-1183.

9. Zmora O, Potenti FM, Wexner SD, et al. Gracilis muscle trans position for iatrogenic rectourethral fistula. Ann Surg. 2013;237(4):483-487.

10. Ganio E, Martina S, Novelli E. Transperineal repair with bulbocavernosus muscle interposition for recto-urethral fistula. Colorectal Dis. 201;15(3):e138-e143.

11. Moretto G, Casaril A, Inama M. Use of biological mesh in trans-anal treatment for recurrent recto-urethral fistula. Int Urol Nephrol. 201; doi: 10.1007/s11255-017-1652-5.

12. Lesser T, Aboseif S, Abbas MA. Combined endorectal advancement flap with Alloderm graft repair of radiation and cryoabaltion-induced rectourethral fistula. Am Surg. 2008;74(4):341-354(5).

13. Gierth M, Dezinger S, Liebig-Horl G, et al. Management of rectourinary fistula after urologicalinterventions using biodesigned mesh: first experiences of an innovative technique. Int J Colorectal Dis. 2015;30(10):1417-1422. 